\title{
p16ink4 and cytokeratin 7 immunostaining in predicting HSIL outcome for low-grade squamous intraepithelial lesions: a case series, literature review and commentary
}

Eric C Huang ${ }^{1}$, Mary M Tomic ${ }^{1}$, Suchanan Hanamornroongruang ${ }^{2}$, Emily E Meserve ${ }^{3}$, Michael Herfs ${ }^{4,5}$ and Christopher P Crum ${ }^{3,5}$

${ }^{1}$ Department of Pathology and Laboratory Medicine, University of California, Davis Medical Center, Sacramento, CA, USA; ${ }^{2}$ Department of Pathology, Siriraj Hospital, Mahidol University, Bangkok, Thailand; ${ }^{3}$ Division of Women's and Perinatal Pathology, Department of Pathology, Brigham and Women's Hospital, Harvard Medical School, Boston, MA, USA and ${ }^{4}$ Laboratory of Experimental Pathology, GIGA-Cancer, University of Liege, Liege, Belgium

p16ink4 and cytokeratin 7 (CK7) have been proposed to identify low-grade squamous intraepithelial lesions (LSIL) at greater or lesser risk for an outcome of high-grade squamous intraepithelial lesion (HSIL). We correlated CK7 and p16ink4 staining in LSILs with outcome on follow-up and placed this information in the context of prior reports. Cervical LSIL biopsies with at least 1-year follow-up information were immunostained for CK7 and p16ink4. Follow-up outcomes included no SIL, LSIL (persistence) or HSIL (CIN2+). In all, 109 LSILs were studied and $18.3 \%$ stained positive for CK7. Ninety-one percent of CK7-negative LSILs regressed, $4.5 \%$ persisted, and $4.5 \%$ had an HSIL outcome, versus 60,20 , and $20 \%$ of CK7-positive LSILs, respectively $(P=0.036)$. p16ink 4 status did not significantly associate with outcome. Review of the literature revealed a highly variable rate of both positive p16ink4 immunoreactivity in LSIL and CIN2+ outcome for p16-positive LSIL but a consistently high negative predictive value ( $>90 \%$ ) in the case of no/low p16 expression. Inter-observer reproducibility for the diagnosis of CIN2 in the literature ranged from poor to good, with unanimous agreement on the diagnosis of CIN2 occurring in less than $25 \%$ of cases. As with high-risk human papillomavirus testing, the most clinically useful result of p16ink4 staining is a negative test, implying no lesion or CIN1 and conferring a low risk of HSIL outcome. HSIL outcomes ('progression') are highly variable and are subject to wide differences in inter-observer interpretation for CIN2. This argues against the wisdom of relying on p16ink4 to both predict CIN2+ or upgrade CIN1 to CIN2. It also begs the question of whether CIN2 should be replaced by an alternate and less pejorative term (SIL of intermediate grade) for lesions that are not reproducibly classified as LSIL or HSIL, with an appropriate management scheme.

Modern Pathology (2016) 29, 1501-1510; doi:10.1038/modpathol.2016.141; published online 12 August 2016

Human papillomavirus (HPV) is strongly linked to cervical cancer and its precursors. HPV vaccination has gained traction as a measure of cancer prevention, but the management of women with abnormal cervical cytology and biopsy proven disease

Correspondence: Dr EC Huang, MD, PhD, Department of Pathology and Laboratory Medicine, University of California, Davis Medical Center, 4400V Street, PATH Building, Sacramento, CA 95817, USA.

E-mail: ecchuang@ucdavis.edu

${ }^{5}$ Drs Herfs and Crum contributed equally as senior authors in this manuscript.

Received 8 June 2015; revised 20 June 2016; accepted 20 June 2016; published online 12 August 2016 remains constrained by complex algorithms that expend considerable resources. One tool that has gained attention recently is p16ink4 (p16), a cyclin-dependent kinase inhibitor that is overexpressed in the presence of high-risk HPVs (hrHPV). ${ }^{1}$ p16 staining is strongly associated with high-grade squamous intraepithelial lesion (HSIL/ CIN2 or CIN3) and in the recently formulated advisory from the Lower Anogenital Squamous Terminology (LAST) standardization project, p16ink4 staining was recommended as a marker to aid in excluding HSIL (when negative) and to adjudicate the diagnosis of precursor lesions that were felt to fall between low-grade squamous intraepithelial 
lesions (LSIL/CIN1) and CIN2. ${ }^{2}$ However, although strong p16 expression is observed in a high percentage of HSILs, it is also expressed in from 20 to $70 \%$ of LSILs, depending on the study. ${ }^{1,3}$ Because of this, the LAST project summary did not recommend that p16 staining be applied to unambiguous LSILs. ${ }^{2}$

The impression from studies of p16 is that this biomarker is a sensitive indicator of HSIL outcome but has lower specificity (reviewed by Sagasta et $a l^{4-6}$ ), similar to hrHPV testing. For this reason, some authors have stressed the negative predictive value of p16 rather than its value in arbitrating a borderline HSIL. ${ }^{7}$

Recently, attention has shifted to another potential biomarker that is highly expressed at the squamocolumnar junction, cytokeratin 7 (CK7). The focus on CK7 has stemmed from its strong expression in a discrete population of cuboidal to low columnar cells near the cervical squamocolumnar junction, in parallel with similar discovery of residual embryonic cells in the esophago-gastric junction. ${ }^{3,8}$ Squamocolumnar junction cells highly express several proteins -including CK7, AGR2, MMP7, and GDA-that are also expressed in cervical cancers. ${ }^{3}$ CK7 has been studied in particular given its already common use in diagnostic pathology. Because most cervical HSILs, adenocarcinomas in situ and squamous cell carcinomas express CK7, they have been presumed to have arisen from squamocolumnar junction cells or closely related progeny. HPV-related lesions located within the mature metaplastic or ectocervical epithelium, as well as the vagina and vulva, are CK7 negative. The implication is that these lesions are not derived from the squamocolumnar junction or emerge in progeny after they have undergone squamous differentiation. Interestingly, the absence of CK7 in these lesions correlates strongest with lower histological grade and, presumably, a lower risk of progression to malignancy. ${ }^{9}$

In a prior pilot study, we showed that CK7-negative LSILs were not followed by an HSIL cytologic or histologic outcome. ${ }^{10}$ Some CK7-positive LSILs were followed by an eventual histologic diagnosis of HSIL, specifically those that generated greater inter-observer disagreement. Thus, we questioned whether LSILs that were negative for CK7 had a low enough HSIL outcome risk to justify using this biomarker to identify lesions that could be followed at a more extended interval. Another group initially found no association between CK7 and HSIL outcome, but on refining their assessment criteria found that CK7-positive LSILs were more likely to be followed by biopsy proven HSIL. ${ }^{11}$ The primary goal of this study was to determine in a new data set the relationship between p16 and CK7 immunostaining in predicting histologic outcomes following biopsy of LSILs. The second was to critically assess this information in context of prior studies, addressing challenges in defining and making a diagnosis of CIN2, perceptions of 'progression', and value of p16 or CK7 in decision making. A recommendation is then made based on these observations.

\section{Materials and methods}

\section{Study Population and Histologic Review}

This study was approved by the institutional review boards at the University of California at Davis (Sacramento, CA, USA) and Brigham and Women's Hospitals (Boston, MA, USA). An institutional retrospective review of all cervical biopsies from 2006 to 2011 by Pathology Laboratory Information System at UC Davis was performed to identified LSIL cases. Clinical follow-up data for cervical cytology, hrHPV status, endocervical curettages, biopsies, and excisions were obtained when available. Consecutive cases that had at least 1-year follow-up and were verified as LSIL by a second observer (CPC) based on published criteria were included in the study. ${ }^{12}$

\section{Immunostaining}

Serial sections from the biopsies were immunostained for CK7 (clone RCK 105, Thermo Scientific, Waltham, MA, USA) and p16 (CINtec, Ventana Medical Systems, Tucson, AZ, USA) and assessed based on previous published criteria. ${ }^{3,10}$ A strong expression of CK7 in multiple contiguous cells in at least the apical layers was classified as positive. Weak diffuse (blush) staining in lower epithelial layers only or in isolated cells in the superficial cell layers was interpreted as negative (Figure 1). Weak p16 staining was considered positive when continuous horizontal nuclear and cytoplasmic staining of at least the basal one-third of the epithelium was seen. Any other staining pattern (usually patchy or focal cells) was interpreted as negative. When the optimal dilution was determined precisely, both CK7 antibodies (clones RCK 105 and OV-TL12/30, Dako, Glostrup, Denmark) displayed a similar immunostaining pattern (data not shown). Representative images of hematoxylin and eosin stain, CK7, and p16-stained cases (positive and negative) are illustrated in Figure 2. Examples of biopsies classified as HSIL outcome are illustrated in Figure 3.

\section{Follow-Up Parameters}

Outcome was designated as:

(1) Regression (negative colposcopic exam and negative cytology including negative hrHPV when available);

(2) Persistence (LSIL on subsequent biopsy or cytology);

(3) HSIL outcome (HSIL on subsequent biopsy or cytology).

The term 'progression' was not used in as much as distinguishing between true progression and either under-sampling of an existing HSIL or the emergence of an independent HSIL could not be done in this study design. Non-diagnostic cervical cytology 

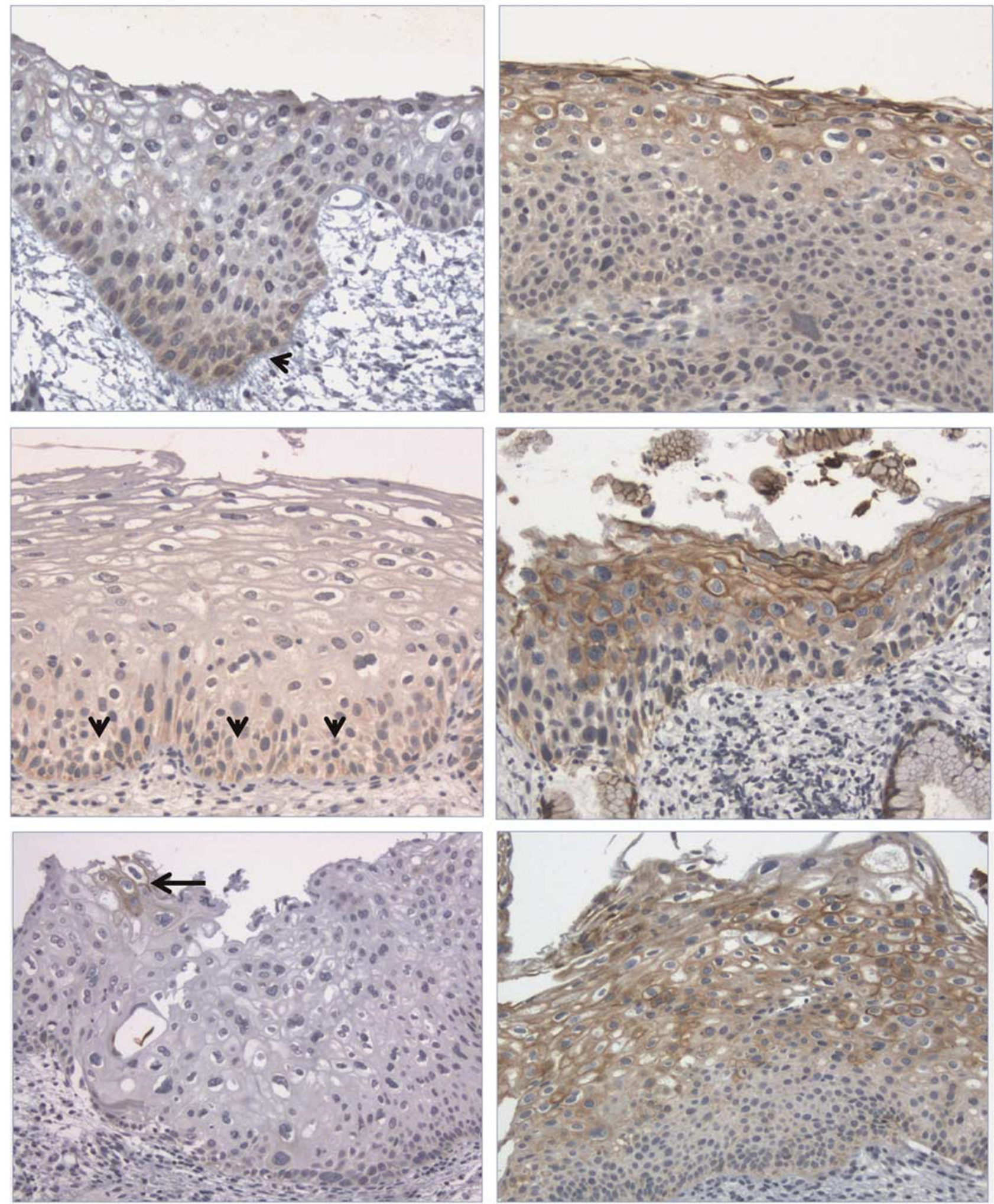

Figure 1 Examples of negative (left column) and positive (right column) staining for CK7 in LSIL. Cases with weak basal or parabasal 'blush' staining (arrowheads) were scored as negative. Moreover, cases with weak focal staining of isolated cells in the superficial cell layer (arrow) were also classified as negative. CK7-positive cases displayed multiple more strongly staining cells, usually in a continuous horizontal arrangement in at least the upper cell layers. 


\section{CK7- LSIL}
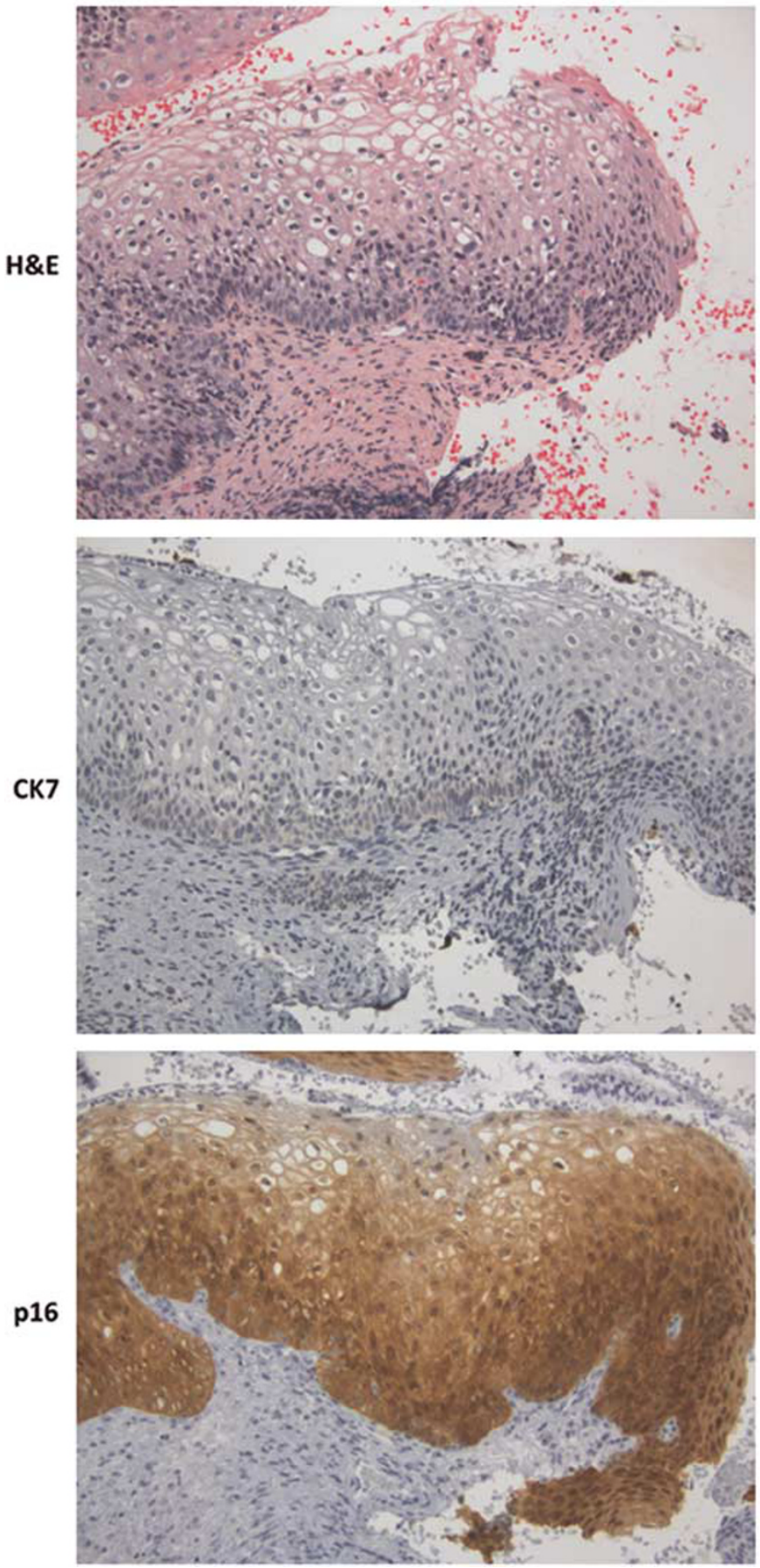

CK7+ LSIL
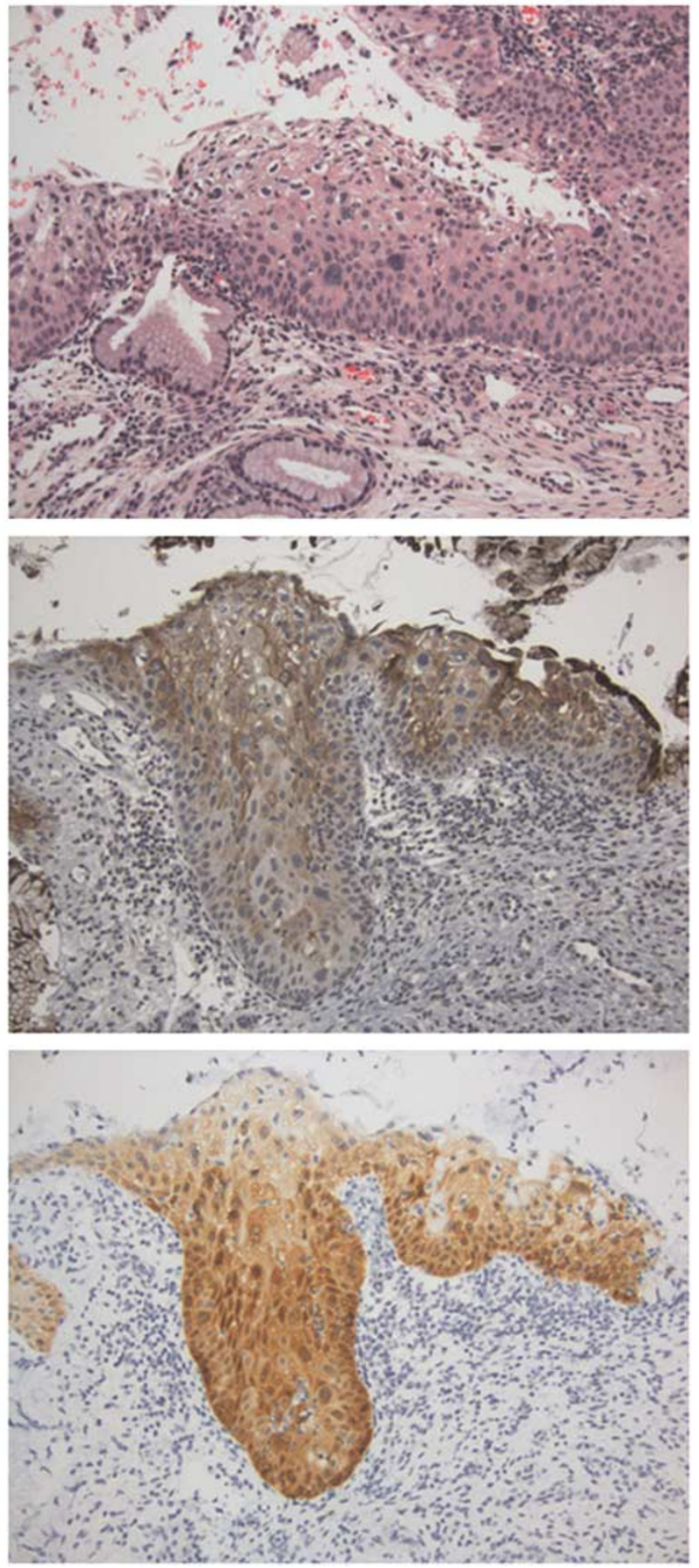

Figure 2 Representative examples of squamoculumnar junction positive (CK7 positive) and negative (CK7 negative) LSILs. The CK7negative LSIL regressed on follow-up, whereas CK7-positive LSIL had an HSIL outcome. Note the strong diffuse p16 staining in both LSILs.

(ASCUS) and negative cytology with positive hrHPV were considered equivocal and not used to subclassify the outcomes.

\section{Statistical Analysis}

Statistical analysis was performed with Fisher's exact test calculator. Differences were considered statistically significant when $P$-values were $<0.05$.

\section{Literature Review}

Based on a review of the literature using search terms in PUBMED such as p16, LSIL, CIN1, outcome, follow-up and inter-observer agreement, a series of prior studies were retrieved that addressed three issues; (1) the degree of inter-observer agreement for a diagnosis of CIN2; (2) the association between p16 and lesion grade; (3) the role of either p16 or CK7 as a 

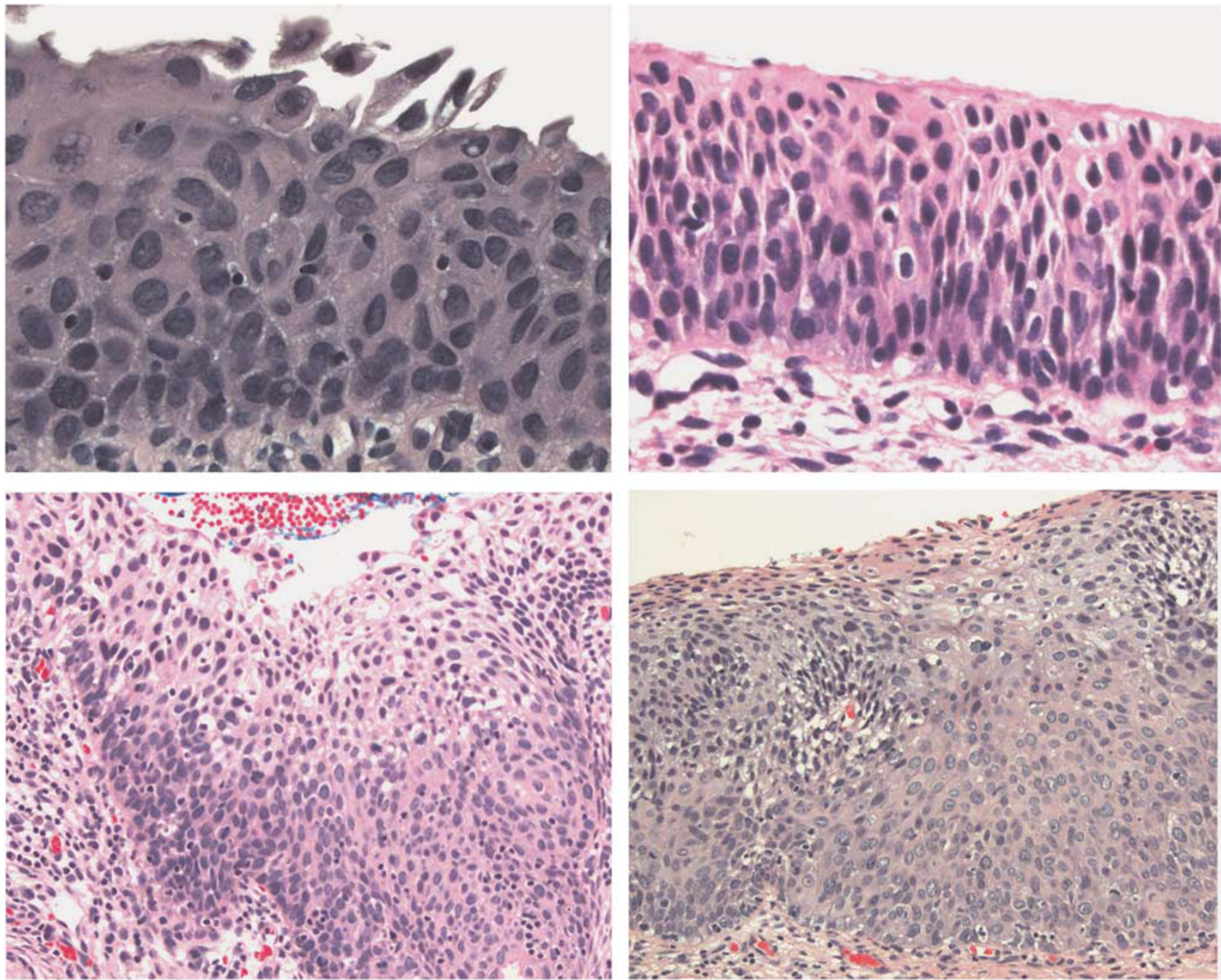

Figure 3 Representative examples of lesions classified as HSIL outcomes. Compare with Figure 2.

Table 1 Clinical outcome of LSILs in respect to CK7 and p16 biomarker expression ( $n(\%))$

\begin{tabular}{|c|c|c|c|c|c|c|c|}
\hline Biomarkers & $\mathrm{n}$ & Regression & Persistence and HSIL outcome & $\mathrm{P}$-value & Regression and persistence & HSIL outcome & $\mathrm{P}$-value \\
\hline CK7 - & 89 & 81 (91.0) & $8(9.0)$ & 0.002 & 85 (95.5) & $4(4.5)$ & 0.036 \\
\hline CK7+ & 20 & $12(60.0)$ & $8(40.0)$ & & $16(80.0)$ & $4(20.0)$ & \\
\hline p16 - & 70 & 63 (90.0) & 7 (10.0) & 0.090 & 66 (94.3) & $4(5.7)$ & 0.453 \\
\hline p16+ & 39 & $30(76.9)$ & 9 (23.1) & & 35 (89.7) & $4(10.3)$ & \\
\hline Total & 109 & 93 (85.3) & $16(14.7)$ & & $101(92.7)$ & $8(7.3)$ & \\
\hline
\end{tabular}

predictor of HSIL (or CIN3) outcome following biopsy of LSIL.

\section{Results}

\section{A Novel Case Series}

Patient characteristics and clinical follow-up data. In all, 109 cases of LSIL confirmed cases with at least 1-year clinical follow-up data were included in the study. Subject ages ranged from 20 to 65 years old (mean $=34.4 \pm 11.2 ;$ median $=31)$. The follow-up interval ranged from 12 to 86.3 months (mean $=32.6$ \pm 19.0 ; median $=27$ ). Regression occurred in $85.3 \%$ of cases $(n=93)$ after initial diagnosis of LSIL, $7.3 \%$ of cases $(n=8)$ had a persistent abnormality, and $7.3 \%$ of cases $(n=8)$ had an HSIL outcome on biopsy or excision (Table 1). The clinical outcome rates in this population of LSIL patients are similar to previously published studies. ${ }^{13-16}$ 
Table 2 Clinical outcome of squamocolumnar junction LSILs further stratified by p16 biomarker status (n (\%))

\begin{tabular}{|c|c|c|c|c|c|c|c|}
\hline Biomarkers & $\mathrm{n}$ & Regression & $\begin{array}{l}\text { Persistence and HSIL } \\
\text { outcome }\end{array}$ & P-value & Regression and persistence & $\begin{array}{c}\text { HSIL } \\
\text { outcome }\end{array}$ & $\mathrm{P}$-value \\
\hline CK7-/p16- & 60 & $57(95.0)$ & $3(5.0)$ & 0.107 & $58(96.7)$ & $2(3.3)$ & 0.594 \\
\hline CK7-/p16+ & 29 & $24(82.8)$ & $5(17.2)$ & & $27(93.1)$ & $2(6.9)$ & \\
\hline $\mathrm{CK} 7+/ \mathrm{p} 16-$ & 10 & $6(60.0)$ & $4(40.0)$ & 1 & $8(80.0)$ & $2(20.0)$ & 1 \\
\hline $\mathrm{CK} 7+/ \mathrm{p} 16+$ & 10 & $6(60.0)$ & $4(40.0)$ & & $8(80.0)$ & $2(20.0)$ & \\
\hline
\end{tabular}

CK7 and p16 immunostains. For CK7 immunostaining, $81.7 \%$ of LSILs (89/109) were negative, whereas $18.3 \%$ of cases (20/109) were positive. Following p16 immunostaining, 64.2\% (70/109) scored negative, and 35.8\% (39/109) scored positive (Table 1).

Correlation of immunostains with clinical follow-up data. For CK7-negative LSILs, the rate of regression, persistent LSIL and HSIL outcome were 91.0\% (81/89), 4.5\% (4/89), and 4.5\% (4/89), respectively. In contrast, LSILs that were CK7 positive had a lower regression rate $(60 \%, 12 / 20)$ and higher rate of persistent LSILs $(20 \%, 4 / 20)$ and HSIL outcome $(20 \%, 4 / 20)$. Statistical analysis showed that CK7negative LSILs were more likely to regress $(P=0.002)$ and CK7-positive LSILs have increased risk of HSIL outcome $(P=0.036)$. In the same patient population, $90.0 \%(63 / 70)$ of p16-negative LSILs regressed, whereas $4.3 \%$ (3/70) persisted and 5.7\% (4/70) had an HSIL outcome. LSILs that were p16-positive regressed, persisted or had an HSIL outcome in $76.9 \%$ (30/39), $12.8 \%$ (5/39), and 10.3\% (4/39) of cases, respectively. Although majority of the p16negative LSILs showed regression, this was not statistically significant $(P=0.090)$. In a similar fashion, p16-positive LSILs did not increase the likelihood of an HSIL outcome $(P=0.453)$. The results are summarized in Table 1.

When LSILs were stratified by combining both p16 and CK7 status, 3\% (2/60) of CK7-negative/p16negative LSILs and $20 \%$ (2/10) of CK7-positive/ p16-positive LSILs had an HSIL outcome. Overall, the addition of p16 did not improve the odds of regression or HSIL outcome in LSILs that have already been risk stratified by CK7 (Table 2).

\section{Literature Review}

Inter-observer agreement for the diagnosis of CIN2. Based on published reports, the inter-observer agreement for the diagnosis of CIN2 is highly variable. ${ }^{17}$ In a study evaluating experienced pathologists, there was a high diagnostic reproducibility in the distinction of CIN2-3 from non-CIN2-3 between pathologists and a series of biopsies with previously determined 'gold standard' diagnoses, with kappa values ranging from 0.779 to 0.887 . However, the non-CIN2-3 group included normal, unsatisfactory, and atypical metaplasia as well as CIN1. Overall, in that study a unanimous verdict (based on independent review) of CIN2 was reached in only $19.4 \%$ of case, and in 31.6 and $47.5 \%$ of CIN1 and CIN3 respectively. ${ }^{18}$ In the ASCUS/LSIL triage study (ALTS), QC reviewers downgraded 29\% of CIN2 to CIN1 or less. ${ }^{19}$ In another study, the diagnosis of CIN2 by initial reviewers was proven highly unreliable, with two study pathologists agreeing on only 13 and $32 \% .^{20}$ In a summary of four studies comparing inter-observer reproducibility for the diagnosis of CIN2, Reuschenbach et al. noted kappa values of 0.22 (poor), 0.41 and 0.44 (fair), and 0.57 (good). They noted a high rate of inter-observer agreement for scoring lesions as p16-positive $(\kappa=0.87-0.91)$ and that by combining p16 and hematoxylin and eosin stain interpretation the kappas ranged from 0.62 to 0.75. The implication was that the presence of p16 positivity facilitated the diagnosis of CIN2. ${ }^{21}$

Association between p16 immunostaining and SIL grade. There is a consistent association in the literature between 'strong' p16 immunoreactivity and either HSIL or the presence of a hrHPV type. Sano et al. noted that over $98 \%$ of hrHPV infections exhibited diffuse p16 staining, usually defined as linear strong staining. Subsequent reports noted positive staining from 64 to $100 \%$ of HSILs, the lesser estimate possibly an artifact created by the lack of a clear biologic distinction between strong and diffuse and strong but focal staining.1,10,22-25 Kong et al. ${ }^{26}$ noted a subset of immature metaplastic atypias that were negative for HPV in situ hybridization also stained strongly for p16. Staining frequencies of LSILs have varied as a function of the frequency of associated low versus hrHPVs. Klaes et $a .^{25}$ and Sano et al. ${ }^{1}$ noted no diffuse staining in low-risk HPV infections or associated LSILs. Klaes et al. observed that $87 \%$ of LSILs associated with hrHPV exhibited diffuse staining. ${ }^{25}$ Herfs et al. noted lower rates of p16 positivity in CK7-negative LSILs versus CK7-positive lesions, but this appears to reflect differences in association with hrHPVs between the two groups. ${ }^{10}$ Overall, approximately a low estimate of one-third to a high estimate of two-thirds of LSILs stain strongly for p16. 10,22-25

Predicting CIN2+ outcome of p16 and/or CK7positive LSIL. Eight reports tracking the histologic outcome of CIN1 cases stained with p16 noted a distinctly higher frequency of CIN2-3 on followup. ${ }^{6,7,27-32}$ In these studies, from 10.3 to $35.4 \%$ of 
p16-positive cases had an HSIL outcome. In contrast, a much narrower range of p16-negative LSILs (0-4.4\%) had an HSIL outcome. Several studies showed the association between p16 and HSIL outcome to be either of arguable significance or not significant. One found no differences in risk, but the HSIL outcome for both groups was inordinately high. ${ }^{33}$ Another found p16 only predictive of an HSIL outcome in the first year of follow-up. ${ }^{34}$ Another found a significant association between p16 and HSIL outcome but it was not corroborated in multivariate analysis. ${ }^{4}$ One study found no significant association between p16 positivity and HSIL outcome. In that study, the HSIL outcome for p16 positive and negative LSILs was $15.0 \%$ and $9.4 \%$ respectively. ${ }^{35}$ In the current study, it was $10.3 \%$ and $5.7 \%$, respectively.

Two notable observations from the literature pertain to inter-study predictions of HSIL outcome from p16 staining and observer agreement. Sagasta et al. summarized sensitivity, specificity, positive, and negative predictive values for p16 staining as a predictor of HSIL outcome based on prior reports. Respective ranges for these parameters were 59-100, 37-72, 10-35, and 90-100. The most consistent finding across all of these studies was the negative predictive value for HSIL outcome of a p16-negative LSIL (90-100). ${ }^{4}$

The referenced studies were reviewed to determine which ones provided histologic illustrations of lesion progression. Virtually all of the studies illustrated sections stained with either p16 or CK7. Only two studies illustrated paired initial (LSIL) and outcome (CIN2-3) images. ${ }^{4,30}$

Only three studies have examined the relationship between CK7 staining of LSIL and HSIL outcome diagnosis. In one, CK7-negative cases with unanimous LSIL agreement had no HSIL outcomes. In contrast, a subset of CK7-positive cases generated more disagreement, with HSIL confirmed on loop electrical excision procedure (LEEP), suggesting that some LSILs at the squamocolumnar junction might be underdiagnosed CIN2. ${ }^{10}$ In the current study, CK7-positive LSILs were found to have a significantly higher likelihood of HSIL outcome. A preliminary study by Paquette et al. did not find a relationship between CK7 positivity and HSIL outcome. However, a follow-up study by the same authors with revised criteria for interpreting CK7 noted a significant association with HSIL. ${ }^{11}$ The associations between CK7 positivity and HSIL outcome in the current study and that of Paquette et al. were both significant but with $P$-values of 0.036 and 0.05. In all, CK7-negative consensus LSILs from three studies had HSIL outcomes of $0 \%, 4.5 \%$, and $11.5 \%$, respectively. ${ }^{10,11}$

\section{Discussion}

Efficient management of preinvasive cervical squamous neoplasia is limited because there is no single biomarker that will effectively segregate precursors that will invariably progress to cancer. The fact that up to two-thirds of CIN2 lesions in young women will disappear spontaneously following biopsy is a testimony to the limitation of histology as a predictor of outcome, at least at the mid to lower end of the precursor spectrum. ${ }^{14,15}$ As might be expected, the presence of carcinogenic HPV is not by itself sufficiently predictive of an HSIL or cancer outcome to merit intervention in most circumstances. ${ }^{5}$ One exception might be persistent hrHPV in older women where tissue sampling may be warranted. ${ }^{36}$ The greatest value of HPV testing is in the triage of cytologic abnormalities and in particular when it is negative, permitting a longer follow-up interval. ${ }^{37}$

The histologic management of patients with cervical abnormalities is more complex because a biomarker (p16) positive result might lead to a diagnosis that prompts a surgical procedure. ${ }^{2}$ Although an HPV-positive ASCUS usually leads only to a colposcopic exam and (if negative) follow-up with an established algorithm, a p16-positive result in a cervical biopsy could lead to upgrading a squamous intraepithelial lesion and result in a LEEP. 2,5,37

Following the introduction of LEEP, management of preinvasive cervical lesions was predicated largely on the histologic distinction between CIN1 and CIN2; the former followed and the latter excised. ${ }^{38}$ A wealth of data from studies reviewed here has shown that the diagnosis of CIN2, connoting HSIL, is variably and often poorly reproduced by multiple observers and in many cases is not consistently distinguished from CIN1. This has resulted in LEEPs for many women whose precursor lesions were erroneously over-classified as CIN2. ${ }^{17}$ The fall-out has been in the form of not only unnecessary surgery but also potential obstetrical complications leading to a greater risk of premature delivery in some women. ${ }^{39}$

p16 immunostaining has been touted as a biomarker to address the conundrum of cervical precursor diagnosis, largely because of its strong association with hrHPV-associated precursor lesions. ${ }^{1}$ Its potential has been examined from three perspectives. The first is the use of p16 to distinguish benign or reactive processes from precursor lesions of any grade associated with hrHPV. The second is its value in separating LSIL from HSIL. The third is its value as a predictor of HSIL outcome. Based on the literature, the value of p16 in distinguishing between SIL and benign conditions is significant, a claim that is corroborated by the high negative predictive value of this biomarker, both in diagnosis and followup. ${ }^{1,27}$ Accordingly, any intraepithelial lesion that is p16 negative is unlikely to be HSIL. Moreover, squamous intraepithelial lesions that are p16 negative are much less likely to have an HSIL outcome. ${ }^{4}$ This spectrum of utility is very similar to that of hrHPV testing, which is particularly decisive in diagnosis or management when it is negative. ${ }^{5}$ p16 can also be useful in improving the reproducibility of 
lesion recognition by assisting the pathologist in reinforcing threshold criteria for the diagnosis of a lesion. ${ }^{40}$ However, based on the high frequency of p16 positivity in LSIL, the highly variable degree inter-observer reproducibility in separating CIN1 from CIN2, and the high regression rate of lesions classified as CIN2, the value of p16 immunostaining in assigning lesion grade in a clinically meaningful way is at best uncertain.

The question from the studies claiming better diagnostic reproducibility with p16 staining is whether the improved reproducibility in recognizing p16 positivity is confused with greater accuracy in recognizing CIN2. There is potential for equating so-called 'cellular transformation'-a term used by some to describe the cellular milieu in which p16 is expressed in HPV infected cells-with either HSIL or a substantial risk of progression to cancer. Moreover, if pathologists assume diffuse p16 staining is tantamount to HSIL and p16 staining is held equivalent to CIN2+, the diagnosis of CIN2 based on p16 staining becomes self-fulfilling. Appropriately, the LAST project has recommended that p16 not be used to evaluate clear-cut LSILs in practice. ${ }^{2}$ However, this provision does not address the fact that the degree to which p16 staining is used will be inversely proportional to the ability of the pathologist to identify clear-cut LSILs and distinguish them from CIN2. Given the degree of inter-observer variation in CIN1 or CIN2 interpretation, it is logical to assume that p16 might be used frequently by practitioners to differentiate the two squamous intraepithelial lesions. In a recent review, Maniar and Nayar observe that the new guidelines from the LAST project include recommendations 'to expand use of the immunohistochemical marker p16 to distinguish between lowgrade squamous intraepithelial lesion and high-grade squamous intraepithelial lesion/intraepithelial neoplasia 2'. ${ }^{40}$ This statement carries with it the implication that practitioners will rely heavily on p16 in their diagnostic practice.

The question addressed in this study was whether p16 or CK7 staining would distinguish LSILs likely to have an HSIL outcome from those that did not. Many studies claim a higher rate of HSIL outcome in p16-positive LSILs and on the surface this is not surprising given the higher association between p16 and HSIL in general. ${ }^{1,10,22-25}$ Nevertheless, the frequency of reported HSIL outcome is markedly variable, ranging from 10 to $40 \%$ in the reports that were reviewed. This wide variability in CIN2 outcome could have several explanations but the most compelling would be variability in the interpretation of CIN2, the most common HSIL outcome in LSIL follow-up studies. From the review of the literature, none of the follow-up studies has illustrated enough examples of CIN2 to ascertain whether the application of criteria for CIN2 were consistent. Moreover, illustrated examples of progression from CIN1 to CIN2 that could be critically reviewed are lacking. We have included examples of biopsies classified by us as HSIL outcomes in Figure 3 and recognize that pathologists who review these images might or might not corroborate the diagnosis of HSIL in some. In a prior study, we found that many outcome diagnoses of CIN2+ were downgraded on review, leading us to believe that actual progression from LSIL to a 'bona-fide' HSIL occurs in $<10 \%$ of LSILs. ${ }^{41}$ It is noteworthy that the more recent reports-including this one-assessing p16 as an independent predictor of HSIL outcome have been less enthusiastic in their conclusions and have not recommended this biomarker due to lack of a significant (or independent) association with HSIL outcome. ${ }^{4,33-35}$

The primary purpose of the current study was not to determine whether CK7 was useful for predicting HSIL outcome. Rather, the intent was to determine whether LSILs not expressing squamocolumnar junction biomarkers would be particularly unlikely to be followed by HSIL. The preliminary study by Herfs et al. suggested that CK7-negative LSILs might have an exceedingly low risk of HSIL outcome and identify-by its absence-a subset of p16-positive/ CK7-negative LSILs that could potentially be followed at longer follow-up interval. ${ }^{10}$ However, the follow-up risk in the study by Paquette et al. ${ }^{11}$ for CK7-negative LSILs (11.5\%) is higher than we expected and the risk in this study is lower but not zero, at $4.5 \%$. Nevertheless, both studies suffer by definition from the fundamental problem of including CIN2 as an HSIL outcome for the reasons previously discussed. Thus, the value of CK7 (or p16) as a useful negative or positive predictor of HSIL outcome will require not only larger studies, but studies that minimize the potential error imposed by equating CIN2 with HSIL. Setting the endpoint at CIN3 rather than CIN2, as proposed by others, is more appropriate. ${ }^{20}$

The lack of clarity regarding the diagnostic criteria for-and significance of-CIN2 begs the question as to whether this diagnosis has any real value in the current management of women with preinvasive cervical neoplasia. ${ }^{20}$ There are three valid reasons for abandoning this diagnostic term. First, there is much greater reproducibility in separating CIN3 from CIN1. ${ }^{20}$ Second, there is a high rate of shortterm resolution for lesions classified as CIN2. ${ }^{14,15}$ Third, there is the recent widespread adoption of the terms LSIL and HSIL to connote lesions with different risks of cancer outcome. ${ }^{2}$ Thus, this is a timely opportunity to consider removing CIN2 from the HSIL category and replacing it with an alternate term. The diagnostic term should reflect the inherent uncertainty of the diagnosis yet be useful in practice. One option is Squamous Intraepithelial Lesion of Intermediate (or Indeterminate) Grade (INSIL). This or a comparable term would provide a more realistic expectation of the pathologists ability to subclassify squamous precursors, would be less pejorative, and might invite a more measured clinical response. 


\section{Acknowledgments}

This study was supported in part by a grant from the National Cancer Institute (5R21CA173190-02 to CPC) and funding from UC Davis Department of Pathology and Laboratory Medicine (ECH). This work was presented in part at the 104th Annual Meeting of the United States and Canadian Academy of Pathology, Boston, MA, USA 2015.

\section{Disclosure/conflict of interest}

The authors declare no conflict of interest.

\section{References}

1 Sano T, Oyama T, Kashiwabara $\mathrm{K}$ et al. Expression status of p16 protein is associated with human papillomavirus oncogenic potential in cervical and genital lesions. Am J Pathol 1998;153:1741-1748.

2 Darragh TM, Colgan TJ, Cox JT et al. The lower anogenital squamous terminology standardization project for HPV-associated lesions: Background and consensus recommendations from the college of American pathologists and the american society for colposcopy and cervical pathology. Arch Pathol Lab Med 2012;136:1266-1297.

3 Herfs M, Yamamoto Y, Laury A et al. A discrete population of squamocolumnar junction cells implicated in the pathogenesis of cervical cancer. Proc Natl Acad Sci USA 2012;109:10516-10521.

4 Sagasta A, Castillo P, Saco A et al. p16 staining has limited value in predicting the outcome of histological low-grade squamous intraepithelial lesions of the cervix. Mod Pathol 2016;29:51-59.

5 The Atypical Squamous Cells of Undetermined Significance/Low-Grade Squamous Intraepithelial Lesions Triage Study (ALTS) Group. Human papillomavirus testing for triage of women with cytologic evidence of low-grade squamous intraepithelial lesions: baseline data from a randomized trial. J Natl Cancer Inst 2000;92:397-402.

6 Nishio S, Fujii T, Nishio H et al. p16(INK4a) immunohistochemistry is a promising biomarker to predict the outcome of low grade cervical intraepithelial neoplasia: comparison study with HPV genotyping. J Gynecol Oncol 2013;24:215-221.

7 Hariri J, Øster A. The negative predictive value of p16INK4a to assess the outcome of cervical intraepithelial neoplasia 1 in the uterine cervix. Int J Gynecol Pathol 2007;26:223-228.

8 Wang X, Ouyang $\mathrm{H}$, Yamamoto $\mathrm{Y}$ et al. Residual embryonic cells as precursors of a Barrett's-like metaplasia. Cell 2011;145:1023-1035.

9 Yang EJ, Quick MC, Hanamornroongruang S et al. Microanatomy of the cervical and anorectal squamocolumnar junctions: a proposed model for anatomical differences in HPV-related cancer risk. Mod Pathol 2015;28:994-1000.

10 Herfs M, Parra-Herran C, Howitt BE et al. Cervical squamocolumnar junction-specific markers define distinct, clinically relevant subsets of low-grade squamous intraepithelial lesions. Am J Surg Pathol 2013;37: 1311-1318.
11 Paquette C, Mills AM, Stoler MH. Predictive value of cytokeratin 7 immunohistochemistry in cervical lowgrade squamous intraepithelial lesion as a marker for risk of progression to a high-grade lesion. Am J Surg Pathol 2016;40:236-243.

12 Nucci MR, Crum CP. Redefining early cervical neoplasia: recent progress. Adv Anat Pathol 2007;14:1-10.

13 Cox JT, Schiffman M, Solomon D. Prospective followup suggests similar risk of subsequent cervical intraepithelial neoplasia grade 2 or 3 among women with cervical intraepithelial neoplasia grade 1 or negative colposcopy and directed biopsy. Am J Obstet Gynecol 2003;188:1406-1412.

14 Moscicki AB, Ma Y, Wibbelsman C et al. Rate of and risks for regression of cervical intraepithelial neoplasia 2 in adolescents and young women. Obstet Gynecol 116:1373-1380.

15 Moscicki AB, Shiboski S, Hills NK et al. Regression of low-grade squamous intra-epithelial lesions in young women. Lancet 2004;364:1678-1683.

16 Ostor AG. Natural history of cervical intraepithelial neoplasia: a critical review. Int J Gynecol Pathol 1993; 12:186-192.

17 Stoler MH, Schiffman M. Interobserver reproducibility of cervical cytologic and histologic interpretations: realistic estimates from the ASCUS-LSIL triage study. JAMA 2001;285:1500-1505.

18 Cai B, Ronnett BM, Stoler $\mathrm{M}$ et al. Longitudinal evaluation of interobserver and intraobserver agreement of cervical intraepithelial neoplasia diagnosis among an experienced panel of gynecologic pathologists. Am J Surg Pathol 2007;31:1854-1860.

19 Castle PE, Stoler MH, Solomon D et al. The relationship of community biopsy-diagnosed cervical intraepithelial neoplasia grade 2 to the quality control pathologyreviewed diagnoses: an ALTS report. Am J Clin Pathol 2007;127:805-815.

20 Carreon JD, Sherman ME, Guillén D et al. CIN2 is a much less reproducible and less valid diagnosis than CIN3: results from a histological review of populationbased cervical samples. Int J Gynecol Pathol 2007;26: 441-446.

21 Reuschenbach M, Wentzensen N, Dijkstra MG et al. p16INK4a immuno-histochemistry in cervical biopsy specimens: a systematic review and meta-analysis of the interobserver agreement. Am J Clin Pathol 2014;142:767-772.

22 Agoff SN, Lin P, Morihara J et al. p16(INK4a) expression correlates with degree of cervical neoplasia: a comparison with Ki-67 expression and detection of high-risk HPV types. Mod Pathol 2003;16:665-673.

23 Tringler B, Gup CJ, Singh $\mathrm{M}$ et al. Evaluation of p16INK4a and pRb expression in cervical squamous and glandular neoplasia. Hum Pathol 2004;35:689-696.

24 Keating JT, Cviko A, Riethdorf S et al. Ki-67, cyclin E, and p16INK4 are complimentary surrogate biomarkers for human papilloma virus-related cervical neoplasia. Am J Surg Pathol 2001;25:884-891.

25 Klaes R, Benner A, Friedrich $\mathrm{T}$ et al. p16INK4a immunohistochemistry improves interobserver agreement in the diagnosis of cervical intraepithelial neoplasia. Am J Surg Pathol 2002;26:1389-1399.

26 Kong CS, Balzer BL, Troxell ML et al. p16INK4A immunohistochemistry is superior to HPV in situ hybridization for the detection of high-risk HPV in atypical squamous metaplasia. Am J Surg Pathol 2007;31:33-43. 
27 Maniar KP, Sanchez B, Paintal A et al. Role of the biomarker p16 in downgrading -CIN 2 diagnoses and predicting higher-grade lesions. Am J Surg Pathol 2015;39:1708-1718.

28 Cortecchia S, Galanti G, Sgadari C et al. Follow-up study of patients with cervical intraepithelial neoplasia grade 1 overexpressing p16Ink4a. Int J Gynecol Cancer 2013;23:1663-1669.

29 Pacchiarotti A, Ferrari F, Bellardini P et al. Prognostic value of $\mathrm{p} 16$-INK4A protein in women with negative or CIN1 histology result: a follow-up study. Int J Cancer 2014;134:897-904.

30 del Pino M, Garcia S, Fusté V et al. Value of p16(INK4a) as a marker of progression/regression in cervical intraepithelial neoplasia grade 1. Am J Obstet Gynecol 2009;201:488 e1-7.

31 Ozaki S, Zen Y, Inoue M. Biomarker expression in cervical intraepithelial neoplasia: potential progression predictive factors for low-grade lesions. Hum Pathol 2011;42:1007-1012.

32 Razmpoosh M, Sansregret A, Oligny LL et al. Assessment of correlation between p16INK4a staining, specific subtype of human papillomavirus, and progression of LSIL/CIN1 lesions: first comparative study. Am J Clin Pathol 2014;142:104-110.

33 Quint KD, de Koning MN, Quint WG et al. Progression of cervical low grade squamous intraepithelial lesions: in search of prognostic biomarkers. Eur J Obstet Gynecol Reprod Biol 2013;170:501-506.

34 Liao GD, Sellors JW, Sun HK et al. p16INK4A immunohistochemical staining and predictive value for progression of cervical intraepithelial neoplasia grade 1 : a prospective study in China. Int J Cancer 2014;134: 1715-1724.

35 Mills AM, Paquette C, Castle PE et al. Risk stratification by p16 immunostaining of CIN1 biopsies: a retrospective study of patients from the quadrivalent HPV vaccine trials. Am J Surg Pathol 2015;39:611-617.

36 Smelov V, Elfstrom KM, Johansson AL et al. Long-term HPV type-specific risks of high-grade cervical intraepithelial lesions: a 14-year follow-up of a randomized primary HPV screening trial. Int J Cancer 2015;136: 1171-1180.

37 Massad LS, Einstein MH, Huh WK et al. 2012 updated consensus guidelines for the management of abnormal cervical cancer screening tests and cancer precursors. Obstet Gynecol 2013;121:829-846.

38 Prendiville W, Cullimore J, Norman S. Large loop excision of the transformation zone (LLETZ). A new method of management for women with cervical intraepithelial neoplasia. Br J Obstet Gynaecol 1989;96: 1054-1060.

39 Samson SL, Bentley JR, Fahey TJ et al. The effect of loop electrosurgical excision procedure on future pregnancy outcome. Obstet Gynecol 2005;105:325-332.

40 Maniar KP, Nayar R. HPV-related squamous neoplasia of the lower anogenital tract: an update and review of recent guidelines. Adv Anat Pathol 2014;21: 341-358. Erratum in: Adv Anat Pathol.

41 Chen EY, Tran A, Raho CJ et al. Histological 'progression' from low (LSIL) to high (HSIL) squamous intraepithelial lesion is an uncommon event and an indication for quality assurance review. Mod Pathol 2010;23:1045-1051. 